\title{
Symbolic Fusion: A Novel Decision Support Algorithm for Sleep Staging Application
}

\author{
Chen $\mathrm{CHEN}^{1,2}$, Xue LIU ${ }^{1}$, Adrien UGON ${ }^{4}$, Xun ZHANG ${ }^{2}$, Amara AMARA ${ }^{2}$, Patrick GARDA ${ }^{1}$, \\ Jean-Gabriel GANASCIA ${ }^{1}$, Dr.Carole PHILIPPE ${ }^{3}$, Andrea PINNA ${ }^{1}$ \\ ${ }^{1}$ Sorbonne Université Pierre et Marie CURIE (Paris 06), UMR 7606, LIP6, Paris, France \\ ${ }^{2}$ Institut Supérieur d'Electronique de Paris, ISEP, Paris, France \\ ${ }^{3}$ AP-HP Hôpital Universitaire Pitié Salpêtrière (Paris), Unité Pathologies du sommeil, Paris, France \\ ${ }^{4}$ Laboratoire d'Informatique Médicale et d'Ingénierie des Connaissances en e-Santé, UMRS 1142, Paris, France \\ chen.chen@lip6.fr, andrea.pinna@lip6.fr
}

\begin{abstract}
With the rapid extension of clinical data and knowledge, decision making becomes a complex task for manual sleep staging. In this process, there is a need for integrating and analyzing information from heterogeneous data sources with high accuracy. This paper proposes a novel decision support algorithm-Symbolic Fusion for sleep staging application. The proposed algorithm provides high accuracy by combining data from heterogeneous sources, like EEG, EOG and EMG. This algorithm is developed for implementation in portable embedded systems for automatic sleep staging at low complexity and cost. The proposed algorithm proved to be an efficient design support method and achieved up to $76 \%$ overall agreement rate on our database of 12 patients.
\end{abstract}

\section{Categories and Subject Descriptors}

I.2 [Artificial Intelligence]: Applications and Expert Systems Medicine and science.

\section{General Terms}

Algorithms.

\section{Keywords}

Symbolic Fusion; decision support; sleep staging; polysomnography (PSG)

\section{INTRODUCTION}

Decision Support Algorithms/Systems (DSA/DSS) are becoming popular and ubiquitous for improving outcomes as well as decreasing cost [1]; they have been widely used in clinical applications, such as diabetes [2], depression [3], heart sound diagnosis [4] and so on. However, many of the existing DSA/DSS have inherent shortcomings [5]: 1) lack of a specific clinical problem; 2) selecting inappropriate method; 3) poor consideration and integration of clinical procedure; and 4) inadequate testing. To overcome these shortcomings, we propose symbolic fusion which can be a direct aid to decision making for clinical applications. In symbolic fusion, heterogeneous signals and human knowledge can be combined to make a composite decision [16]. In this paper, symbolic fusion focuses on dealing with a specific clinical problem, i.e. automatic classification of sleep stages. Our proposed method also integrates the full clinical sleep staging procedure, and tested with 12 patient's data.

More than $10 \%$ of the people are seriously affected by sleep disorders, such as sleep apnea and insomnia [6]. These disorders can cause daytime sleepiness, irritability, anxiety and depression [7]. Sleep staging plays an important role as a fundamental step in sleep studies for diagnosis and treatment of sleep disorders. Currently, clinical sleep staging is based on an overnight polysomnography (PSG) recording including electroencephalography (EEG), electrocardiography (ECG), electrooculography (EOG), electromyography (EMG), respiratory effort, blood oxygen saturation and manually score according to the R\&K [8] or AASM manuals [9]. Manual sleep staging has several limitations: 1) it is a time consuming and labor intensive task involving interpretation of different clinical data; 2) interrater reliability concerns exist due to subjective interpretation and decision by physicians. To overcome these limitations, several decision support algorithms have been proposed to realize automatic sleep staging.

One of the most widely used algorithms is Decision Tree [7, 10]. However, it has inherent limitations such as the accuracy is extremely sensitive to small perturbations, which is not suitable for sleep staging because of individual variability in PSG signals. For Artificial Neural Network (ANN) [11-13] and Fuzzy Inference [14], large set of training data is required and the performance depends mostly on the quality of the used feature set. Two important factors which are not considered in Decision Tree, ANN and Fuzzy Inference. Firstly, classifying sleep stages is often thought as an independent classification problem; whereas sleep staging is a time dependent classification problem, which can be influenced by the previous sleep stage and can influence the next sleep stage. Secondly, they use smaller number of signals to reduce the dimension of the feature set. However, to increase the accuracy and reliability of the sleep staging, there is a need to include more signals in the analysis [15].

In this paper, we present symbolic fusion which simulates decision-making process of clinical sleep staging and capable of the following features: 1) it integrates data from heterogeneous sources, like EEG, EMG and EOG which can provide enhanced and complementary decision in comparison to signal data based methods; 2) it can deduce a composite decision because it is based on the cooperation between engineers and clinical experts; 3) it can consider time contextual information effect. 
The aim of the present study is to propose a new decision support algorithm and to use it for a clinical application. Proposed method is used for automatic classification of sleep stages. Due to its scalability and less complexity, it can be easily implemented in embedded systems for assisting doctors to analyze sleep stages. This paper is organized as follows. Subjects and methodology are discussed in Sections 2. Results and discussion are presented in Section 3, followed by conclusions with future directions in Section 4.

\section{SUBJECTS AND METHODOLOGY 2.1 Subjects and Data Acquisition}

Overnight PSG signals were recorded by clinical experts in Hôptial-Tenon (AP-HP) from 12 subjects ( 2 males and 10 females) ranging from 26 to 67 years old (mean=53.42 years, $\mathrm{STD}=14$ years). Healthy subjects and subjects with sleep disorders are included in this study. PSG recordings which involves three EEG channels (C3-A2, C4-A1, O1-A2), two EOG channels (EOG-L, EOG-R) and one chin EMG channel were segmented into 30-s epoch and manually scored into Stage Awake, Stage Rapid-EyeMovement (Stage REM) and three Non-Rapid-Eye-Movement Stages (N1, N2, N3) by experts according to AASM manual. Total 15408 epochs were analyzed and used as a database.

\subsection{Methodology}

The complete sleep staging design flow is illustrated in Figure 1. It consists of three main parts: Pre-processing and Segmentation, Symbolic Fusion and Smoothing.

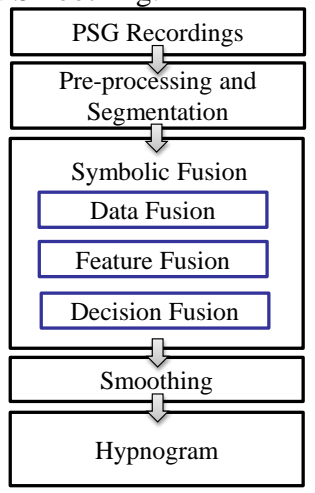

Figure 1. Design flow of sleep staging

\subsubsection{Pre-processing and Segmentation}

Pre-processing is designed to eliminate noise and artifacts. A Butterworth bandpass filter with a cutoff frequency of $0.2-30 \mathrm{~Hz}$ is designed for filtering EEG and EOG signals, and another Butterworth bandpass filter with the cutoff frequency of 5-100 Hz is designed for EMG signal. PSG recordings are segmented into 30 -s epochs after pre-processing.

\subsubsection{Symbolic Fusion}

Fusion is a hierarchical process, starting from raw data processing and goes up-to high level symbolic interpretation. The paradigm "signal-to-symbols" is widely used in many applications, like speech recognition and computer vision [16].

In this paper, we presented symbolic fusion with three-level architecture: data fusion, feature fusion and decision fusion by using paradigm from fusion. Figure 2. presents a detailed flow of symbolic fusion including all the parameters of each level.

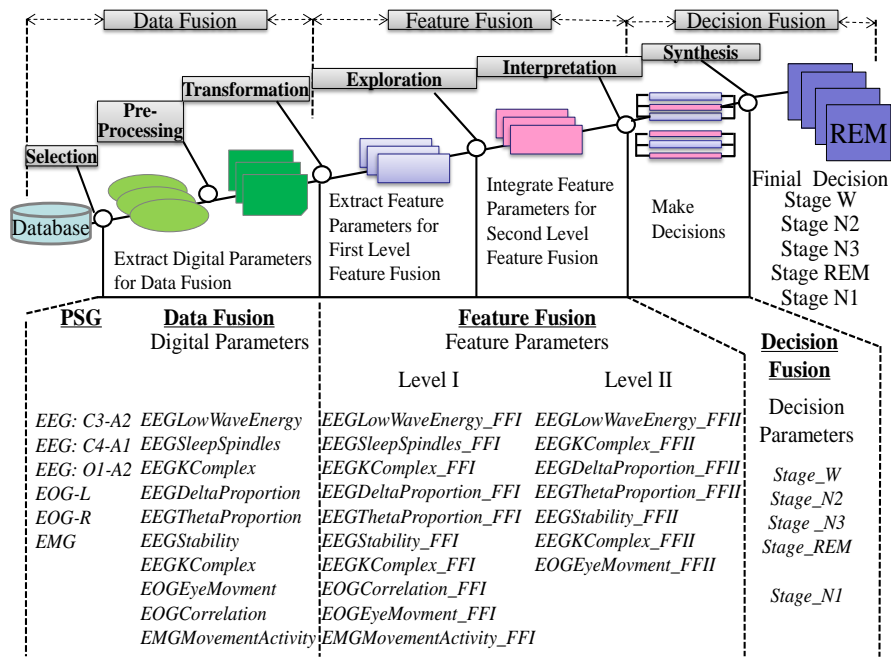

Figure 2. Symbolic fusion design flow

\section{Data Fusion}

It maximizes useful information and minimizes the remaining noise and artifacts. Nine digital parameters are extracted in data fusion. Six parameters are extracted from three different EEG channels; two parameters are extracted from two EOG channels and one parameter is extracted from EMG. Brief description of each parameter is introduced in this section.

$>$ EEGLowWaveEnergy: it represents the energy of the slow wave having frequency between $0.2 \mathrm{~Hz}$ to $2 \mathrm{~Hz}$ in EEG signal.

$>$ EEGSleepSpindles: sleep Spindle is a train of distinct waves having frequency of $11-16 \mathrm{~Hz}$ with duration more than 0.5 seconds. It is used as a main indicator for stage N2. Short Time Fourier Transform (STFT) is used for calculating EEGSleepSpindles.

$>$ EEGKComplex: K-Complex is a well-delineated negative sharp wave immediately followed by a positive component standing out from the background EEG signal, with total duration $\geqslant 0.5 \mathrm{sec}$ [9] which is also a significant indicator to classify Stage N2. Teager Energy Operator (TEO) method is used to calculate K-Complex.

$>$ EEGDeltaProportion: it signifies the power ratio of Delta frequency band (frequency smaller than $2 \mathrm{~Hz}$ ) to the total power of each 30-s epoch.

$>$ EEGThetaProportion: it signifies the power ratio of Theta frequency band (frequency between $4 \mathrm{~Hz}$ and $7 \mathrm{~Hz}$ ) to the total power of each 30-s epoch.

$>$ EEGStability: it signifies the power ratio of the fast wave (frequency more than $18 \mathrm{~Hz}$ ) to the total power. The fast wave is one characteristics of Stage Awake.

$>$ EOGEyeMovment: it represents the number of times eye moves during sleep. The algorithm we used to calculate this parameter is based on [17]. This parameter is used as a main indicator for distinguishing between REM and NREM stage.

$>$ EOGCorrelation: it is the correlation between the left and right eye movements. It indicates whether the movement of the two eyes is conjugate or not.

$>$ EMGMovementActivity: mean absolute value of the EMG signal is calculated as EMGMovementActivity. This parameter is used to indicate the activity level of EMG, which can be used as an indicator of the muscle tone movement in sleep staging. 


\section{Feature Fusion}

Feature fusion is used to transform digital parameters into feature parameters. It simplifies the interpretation of digital parameters, and also performs reduction, matching and normalization of digital parameter sets.

In feature fusion, 2-level fusion is performed. In first level, 9 digital parameters are transferred into feature parameters; in second level, it integrates either 3 EEG channels or 2 EOG channels. e.g., at feature fusion level, 'EEGStability' digital parameter is fused into a 'EEGStabilityFFII' feature parameter which indicates three feature states: Stable, Unstable and Not Confident.

\section{Decision Fusion}

In decision fusion, inference method is used to fulfill specific task on the basis of feature parameters. In order to generate a composite decision of sleep staging, a set of rules were defined under the cooperation between clinical experts and engineers under the guidance of AASM manual. Figure 3. shows a design flow for classification of Stage N2.

\subsection{Smoothing}

After symbolic fusion, a smoothing function is proposed to consider the temporal effects of sleep staging process, and to detect and correct falsely detected sleep transitions, like the transition from Stage Awake to Stage N3.

Due to time dependence of sleep staging process, temporal contextual information based considerations are also included in smoothing process according to AASM manual. At the same time, an error correction is performed in smoothing to detect and correct false sleep transitions.

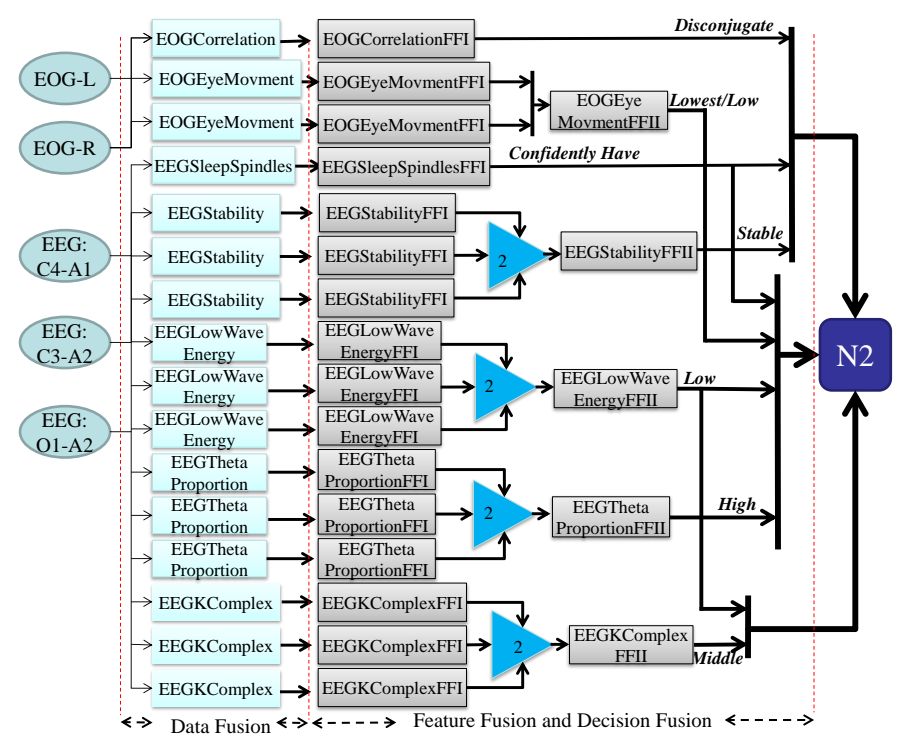

Figure 3. Classification of stage N2

\section{RESULTS AND DISCUSSION}

In this study, Agreement Rate (AR) is used to assess the performance of symbolic fusion. Figure 4. shows the hypnograms of one subject including the comparison between symbolic fusion sleep staging and manual sleep staging from clinical experts. Achieved AR between symbolic fusion and manual sleep staging is approximately $90 \%$ for this patient.

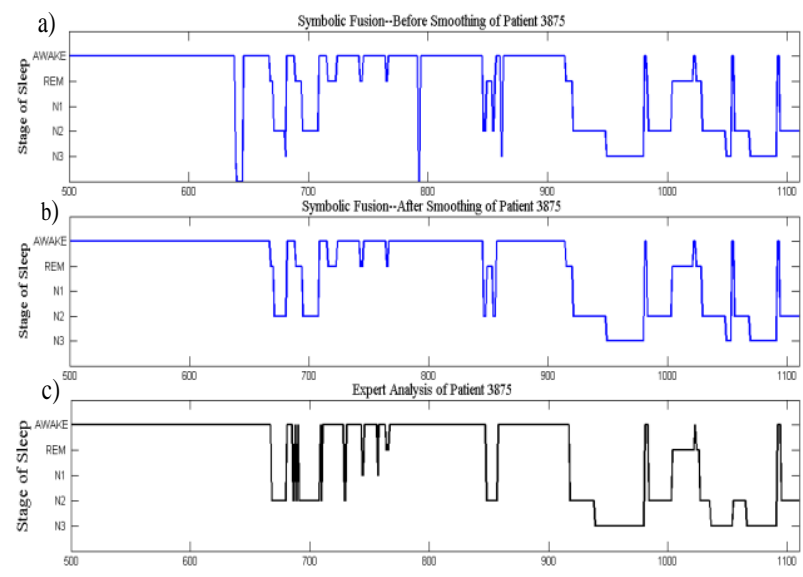

Figure 4. Hypnograms comparison between symbolic fusion and visually analysis: a) automatic staging without smoothing hypnogram using symbolic fusion, b) automatic staging with smoothing hypnogram using symbolic fusion and c) the original manually scored hypnogram.

On our database, overall $76 \%$ AR is achieved for symbolic fusion, which is better in comparison to other decision support algorithms, as reported in $[10,18,19]$. It proved to be an effective method in comparison to decision tree method, which has $\mathrm{AR}$ up to $70 \%$ [10]. In [18], authors reported $61 \%$ AR by using Linear Discriminant Analysis. In [19], authors reported AR of 55.88\%, by using K-Nearest Neighbor method. Proposed method shows the same AR as in [12]. However, in [12], the method is only tested on a healthy subject.

Table 1 shows the comparison with a recent report in literature [14], which used Fuzzy Inference. Our method showed 4.38\%, $2.86 \%, 16.55 \%$ and $3.26 \%$ better AR in Stage Awake, N2, N3 and REM, respectively. It should be noted that AR of Stage N1 doesn't be evaluated. This is because Stage N1 is a transition between awake and asleep which only accounts for $2 \%$ of the total sleep time [20]. Our current work mainly focuses on classifying the others stages. Rules will be added after cooperation between clinical experts and engineers to classify Stage N1 in our further work.

In this paper, it is worth to mention that the present study consists of variety of subjects (i.e., male/female, apnea/non-apnea, and young/old), contrary to most of the studies which only consider healthy subjects $[10,12]$.

Table 1. Agreement Rate comparison between symbolic fusion and other method

\begin{tabular}{|c|c|c|}
\hline & $\begin{array}{c}\text { Symbolic } \\
\text { Fusion }\end{array}$ & $\begin{array}{c}\text { Fuzzy } \\
\text { Inference [14] }\end{array}$ \\
\hline Stage Awake & $89.38 \%$ & $85 \%$ \\
\hline Stage N1 & $\begin{array}{c}\text { Not } \\
\text { Evaluate }\end{array}$ & $18 \%$ \\
\hline Stage N2 & $76.86 \%$ & $74 \%$ \\
\hline Stage N3 & $79.55 \%$ & $63 \%$ \\
\hline Stage REM & $70.26 \%$ & $67 \%$ \\
\hline
\end{tabular}




\section{CONCLUSION AND PERSPECTIVE}

In this study, we proposed a new symbolic fusion based decision support algorithm to realize automatic sleep staging. It can simulate decision making process of clinical sleep staging by integrating data from heterogeneous sources and human knowledge from experts. It provides objective information to improve overall accuracy and simplifies the sleep staging process in comparison to other methods. Proposed algorithm is an efficient method for automatic sleep staging and we achieved an overall agreement rate of $76 \%$ for our database. Due to simple computation and extensibility, this algorithm can be easily implemented in embedded device for remote sleep staging at low cost. Contrary to most of the studies which only consider healthy subjects, our study consists of variety of subjects. In our further work, specific rules will be proposed to realize classification of Stage N1. With this, more subjects will also be involved.

\section{ACKNOWLEDGMENTS}

This work is done under collaboration of Laboratoire d'Informatique de Paris 6 and Institut Supérieur d'Electronique de Paris. We would also like to thank Dr. C. Philippe for the collaboration and for providing the database and help in the field of medicine. This work is also supported by China Scholarship Council (CSC).

\section{REFERENCES}

[1] Fillmore, C.L., Bray, B.E. and Kawamoto, K. 2013. Systematic review of clinical decision support interventions with potential for inpatient cost reduction. BMC medical informatics and decision making. 13, (2013), 135.

[2] Aishwarya S. and Anto S. 2014. A Medical Decision Support System based on Genetic Algorithm and Least Square Support Vector Machine for Diabetes Disease Diagnosis. International Journal of Engineering Sciences \& Research Technology, (2014), 4042-4046.

[3] Trivedi, M.H., Kern, J.K., Grannemann, B.D., Altshuler, K.Z. and Sunderajan, P. 2004. A computerized clinical decision support system as a means of implementing depression guidelines. Psychiatric Services (Washington, D.C.). 55, 8 (Aug. 2004), 879-885.

[4] Stasis, A.C., Loukis, E.N., Pavlopoulos, S.A. and Koutsouris, D. 2003. Using decision tree algorithms as a basis for a heart sound diagnosis decision support system. 4th International IEEE EMBS Special Topic Conference on Information Technology Applications in Biomedicine, 2003 (Apr. 2003), 354-357.

[5] Eberhardt, J., Bilchik, A. and Stojadinovic, A. 2012. Clinical decision support systems: potential with pitfalls. Journal of Surgical Oncology. 105, 5 (Apr. 2012), 502-510.

[6] Ram, S., Seirawan, H., Kumar, S.K.S. and Clark, G.T. 2010. Prevalence and impact of sleep disorders and sleep habits in the United States. Sleep \& Breathing = Schlaf \& Atmung. 14, 1 (Feb. 2010), 63-70.

[7] Liang, S.-F., Kuo, C.-E., Hu, Y.-H. and Cheng, Y.-S. 2012. A rule-based automatic sleep staging method. Journal of Neuroscience Methods. 205, 1 (Mar. 2012), 169-176.

[8] Allan Hobson, J. 1969. A manual of standardized terminology, techniques and scoring system for sleep stages of human subjects. Clinical Neurophysiology. 26, 6 (Jun. 1969), 644.

[9] Conrad Iber, Sonia Ancoli-Israel, Andrew L.Chesson JR, Stuart F.Quan, AASM Manual for the Scoring of Sleep and Associated Events: http://www.aasmnet.org/scoringmanual/. Accessed: 2015-07-01.

[10] Hanaoka, M., Kobayashi, M. and Yamazaki, H. 2001. Automated sleep stage scoring by decision tree learning. Proceedings of the 23rd Annual International Conference of the IEEE Engineering in Medicine and Biology Society, 2001 (2001), 1751-1754 vol.2.

[11] Ebrahimi, F., Mikaeili, M., Estrada, E. and Nazeran, H. 2008. Automatic sleep stage classification based on EEG signals by using neural networks and wavelet packet coefficients. Conference proceedings:Annual International Conference of the IEEE Engineering in Medicine and Biology Society. IEEE Engineering in Medicine and Biology Society. Annual Conference. 2008, (2008), 1151-1154.

[12] Kerkeni, N., Alexandre, F., Bedoui, M.H., Bougrain, L. and Dogui, M. 2005. Automatic Classification of Sleep Stages on a EEG Signal by Artificial Neural Networks. Proceedings of the 5th WSEAS International Conference on Signal, Speech and Image Processing (Stevens Point, Wisconsin, USA, 2005), 128-131.

[13] Tagluk, M.E., Sezgin, N. and Akin, M. 2010. Estimation of sleep stages by an artificial neural network employing EEG, EMG and EOG. Journal of Medical Systems. 34, 4 (Aug. 2010), 717-725.

[14] Álvarez-Estévez, D., Fernández-Pastoriza, J.M., HernándezPereira, E. and Moret-Bonillo, V. 2013. A method for the automatic analysis of the sleep macrostructure in continuum. Expert Systems with Applications. 40, 5 (Apr. 2013), 17961803.

[15] Kushida, C.A. et al. 2005. Practice parameters for the indications for polysomnography and related procedures: an update for 2005. Sleep. 28, 4 (Apr. 2005), 499-521.

[16] Haton, J.-P., Haton, M.-C. and Charpillet, F. 1998. Numeric/Symbolic Approaches for Data and Information Fusion. (1998), 8 p.

[17] Merino, M., Rivera, O., Gomez, I., Molina, A. and Dorronzoro, E. 2010. A Method of EOG Signal Processing to Detect the Direction of Eye Movements. 2010 First International Conference on Sensor Device Technologies and Applications (SENSORDEVICES) (Jul. 2010), 100-105.

[18] Figueroa Helland, V.C., Gapelyuk, A., Suhrbier, A., Riedl, M., Penzel, T., Kurths, J. and Wessel, N. 2010. Investigation of an automatic sleep stage classification by means of multiscorer hypnogram. Methods of Information in Medicine. 49, 5 (2010), 467-472.

[19] Güneş, S., Polat, K. and Yosunkaya, Ş. 2010. Efficient sleep stage recognition system based on EEG signal using k-means clustering based feature weighting. Expert Systems with Applications. 37, 12 (Dec. 2010), 7922-7928.

[20] M Suzanne Stevens, Normal Sleep, Sleep Physiology and Sleep Deprivation, Forman, G. 2003. An extensive empirical study of feature selection metrics for text classification. $J$. Mach. Learn. Res. 3 (Mar. 2003), 1289-130. 